CASE REPORT

\title{
A BRODIE'S ABSCESS OF FEMORAL NECK MIMICKING OSTEOID OSTEOMA: DIAGNOSTIC APPROACH AND MANAGEMENT STRATEGY
}

\author{
Pranshu Agrawal ${ }^{1}$, Anshul Sobti ${ }^{1}$
}

ABSTRACT

BACKGROUND: Brodie's abscess usually presents as a diagnostic dilemma resulting in a diagnostic delay. It mimics various benign and malignant conditions both clinically and radiologically.

CASE DETAILS: This report describes a case of a 14 year old boy who presented with a clinical and radiological picture of osteoid osteoma of proximal femur but found to have a brodie's abscess on histology and culture.

CONCLUSION: It describes the clinical spectrum of subacute osteomyelitis, its unusual MRI features and the importance of obtaining a histological diagnosis before definitive treatment.

KEYWORDS: Brodie's abscess; osteoid osteoma; bone tumour mimickers

DOI: http://dx.doi.org/10.4314/ejhs.v25i4.13

\section{INTRODUCTION}

Brodie's abscess usually presents as a diagnostic dilemma and clinically mimics various benign and malignant conditions (1-3). Also, sometimes the radiological features are misleading (1-5). Brodie's abscess or primary subacute osteomyelitis is known to occur more commonly in children. Metaphyseal lesions are the most common and occur most commonly in proximal tibia $(2,6,7)$. The neck and the surrounding metaphyseal region of proximal femur is a less commonly affected site $(8,9)$. Roberts et al described a classification of brodie's abscess depending on their anatomical location and their resemblance to specific neoplasms. Type $1 \mathrm{~b}$ represents a metaphyseal lesion comprising a radiolucent area with a sclerotic margin along with periosteal reaction (10). This type of lesion closely resembles osteoid osteoma both clinically and radiologically. This report describes a case of a 14 year old boy who presented with a clinical and radiological picture of osteoid osteoma of proximal femur but found to have a brodie's abscess on histology and culture. It describes the clinical spectrum of subacute osteomyelitis, its unusual MRI features and the importance of obtaining a histological diagnosis before treatment.

\section{CASE REPORT}

We report a case of a 14 years old boy who came to our clinic, i.e. the Out-patient Department of a tertiary care referral center in India, in February 2014, with complaints of pain in his left hip. The pain was insidious in onset, dull aching in nature, mild to moderate in severity, more during the nights and was present for the last three months. Physically, he was able to carry out all his daily activities including squatting with some discomfort but was not able to carry out strenuous activities like running and jumping. The patient did not complain of any other constitutional symptoms like fever, weight loss, decreased appetite or night sweats.

On clinical examination, the patient had tenderness anteriorly over the left hip joint, with no local signs of infection. The patient did not have any fixed deformities on the affected site. Passive movements over the left hip, specifically flexion, adduction and internal rotation were restricted and painful terminally as

\footnotetext{
${ }^{1}$ Department of Orthopaedics, Hinduja National Hospital \& Medical Research Centre, , Mahim, Mumbai

Corresponding Author: Pranshu Agrawal ${ }^{1}$, Email: pranshuorthodoc@gmail.com
} 
compared to the right side. There was no limb length discrepancy; however, the patient walked with an antalgic limp. The examination of the right hip, the left knee and the spine showed no positive findings.

The patient was subjected to routine biochemical and radiological investigations. It included a total WBC count of 8,400 , differential neutrophil count of $70 \%$ and lymphocyte count of 26\%. The ESR (erythrocyte sedimentation rate) after 1 hour was 26 and CRP (C- Reactive protein) was $2.8 \mathrm{mg} / \mathrm{L}$. A true size plain radiograph AP view revealed an osteolytic area of around $1 \mathrm{~cm}$ diameter in the calcar region surrounded by osteosclerotic margins along with periosteal reaction along the medial aspect of neck and metaphysis (Figure 1).

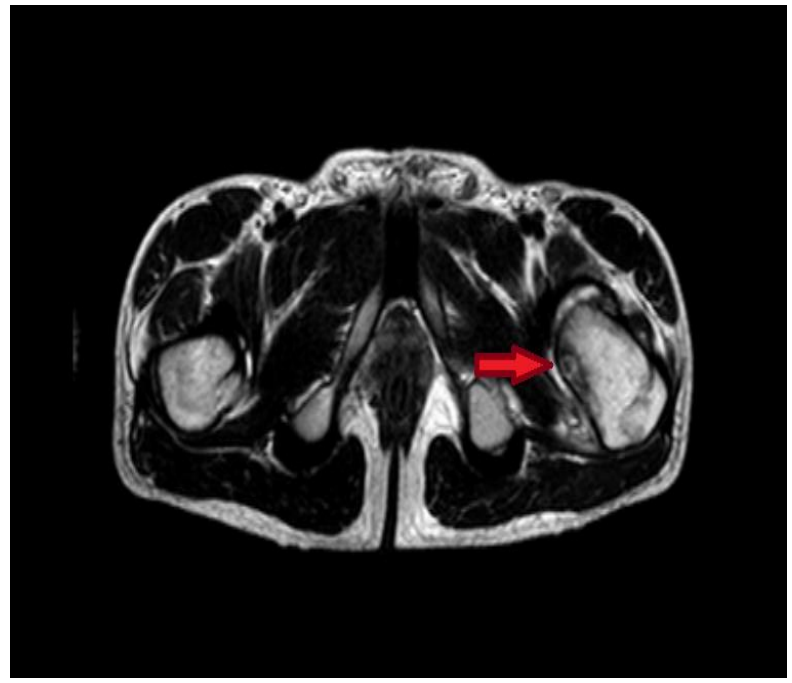

Figure 1: A true size AP radiograph of a 14 year old male with a radiolucent lesion in the calcar region of the left proximal femur with surrounding sclerosis and periosteal reaction

After thorough clinical examination and routine investigations, a provisional diagnosis of an osteoid osteoma was considered, and the patient was further subjected to Magnetic Resonance Imaging (MRI) to substantiate this. MR imaging showed a lesion measuring around $12 \times 8 \mathrm{~mm}$ with a central nidus which was hypointense on T1weighted images and was hyperintense on $\mathrm{T}$ weighted images (Figures. $2 \& 3$ ). The clinical and radiological picture was quite confirmatory for the diagnosis of osteoid osteoma, and it was imperative to treat it with Radio Frequency Ablation. However, the senior surgeon stressed confirming the diagnosis histologically prior to it. Thus, the patient underwent a curettage procedure, which was done through a lateral approach. Under image guidance, a $2 \mathrm{~mm} \mathrm{k}$-wire was directed towards the osteolytic lesion over which a $5 \mathrm{~mm}$ cannulated drill was passed and the cavitary lesion was opened (Figure 4). The track in the outer femoral cortex was widened, and a curette was passed through the tract into the lesion, and the lesion was curetted out and the tissue obtained was sent for histopathology and microbial culture. The histopathology revealed granulation tissue with both acute and chronic inflammatory cells with areas of dead bony tissue, and the culture grew Staphylococcus aureus. These findings led to the final diagnosis of brodie's abscess in contrast to our provisional diagnosis, which was based on clinical examination and radiological features, emphasizing the importance of culture and histology of every suspected bony neoplasm. The patient was managed with antibiotics based on the sensitivity, and he was allowed only partial weight bearing with support for the next 6 weeks. At 6 months' followup, the patient was asymptomatic and could do all the activities which were restricted previously. A MRI scan was repeated at 6 months followup, which did not show any signs of persistent disease.

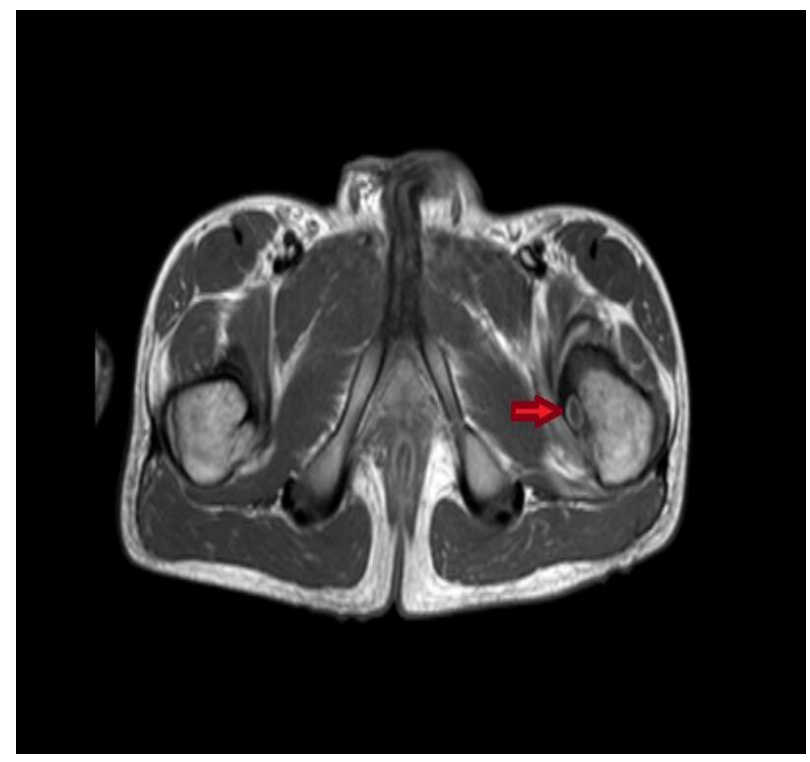

Figure 2: T1 weighted axial MR image showing a hypointense nidus surrounded by a zone of hyperintensity and bone oedema 


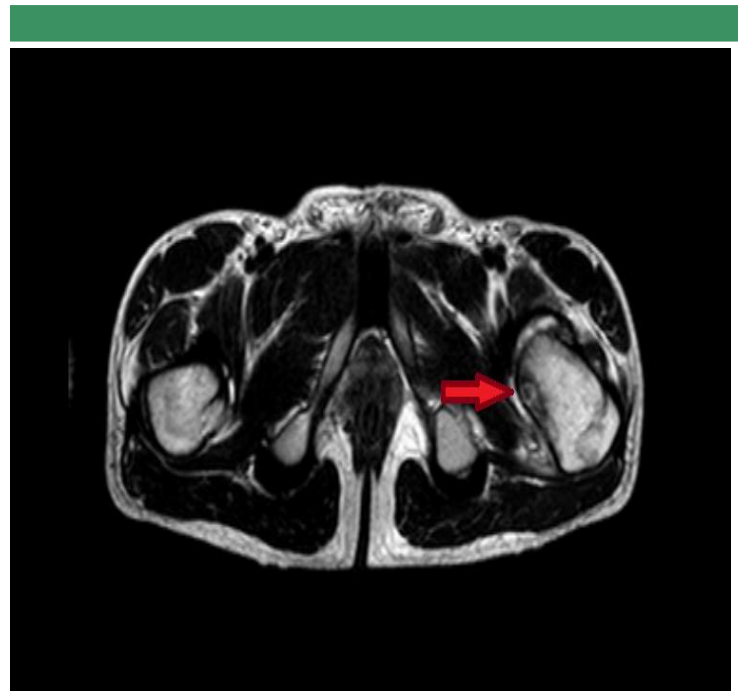

Figure 3: T2 weighted axial image showing the incresed intensity of the nidus as compared to T1 image

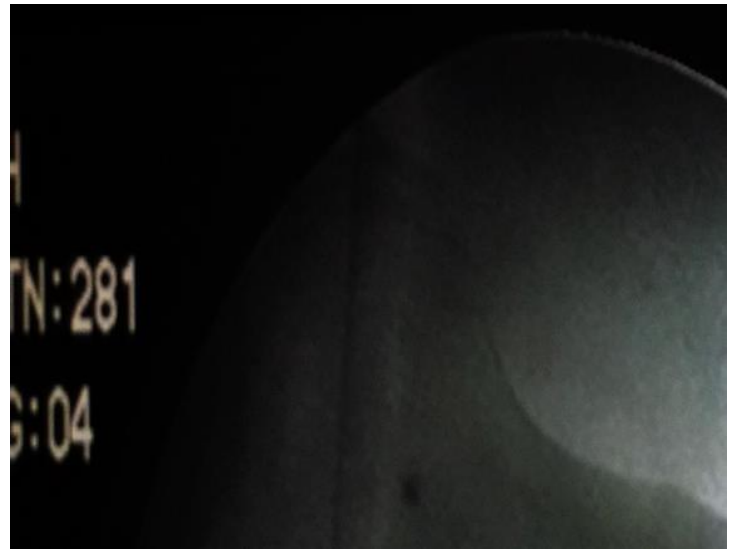

Figure 4: Intra-operative lateral radiograph showing the $k$-wire placed in the lesion opening the cavity

\section{DISCUSSION}

Subacute osteomyelitis has not been strictly defined, but most people agree to its definition as any infectious process in the bony tissue persisting for more than 2 weeks without any symptoms of acute illness $(5,6)$. The infection persists in subacute form either due to low virulence of the organism or good host resistance or because of inappropriate use of antibiotics. The disease occurring due to two earlier reasons is termed as primary subacute osteomyelitis, and is mostly due to haematogenous seeding (5).

Occurring predominantly in young adult males with a history of trauma, recurrent pain and localized tenderness are common presenting symptoms, and are usually associated with fever, chills or malaise due to systemic infection $(5,6)$. However, in a significant number of patients, localized pain or vague symptoms ranging from 1 month to 3 years are the only manifestations of a Brodie's abscess. Radiographically, brodie's abscess appears as a radiolucent lesion, usually eccentric, varying in size from as small as $1 \mathrm{~cm}$ to as large as occupying the entire diameter of the bone, with marginal sclerosis and some periosteal thickening (8). On computed tomography, a sinus tract can be visualized in a few cases. A "penumbra sign" has been described on T1weighted MRI in cases of brodie's abscess. It is a discrete zone of transition of relatively hyperintense signal between the intermediate to low signal intensity abscess cavity and the adjacent oedematous or sclerotic bone marrow on unenhanced T1-weighted imaging. Brodie's abscess is known to mimic osteoid osteoma, more so in the metaphysis of tibia or femur (1-3). Only a few cases have been reported which describe the diagnostic dilemma in the neck of femur (7). The case reported in this paper talks about a brodie's abscess in the neck of femur, specifically in the calcar region which masquerades an osteoid osteoma.

Osteoid osteoma occurs most commonly in the second decade and presents a typical history as was in this case.(1-3, 5,6). Radiographically, a typical osteoid osteoma appears as a dense sclerotic area surrounding a radiolucent nidus and does not usually exceed a size of $15 \mathrm{~mm}$. On computed tomography, the nidus is of low attenuation surrounded by an area of high attenuation. No sinus tracts or cloaca are seen. On MRI T1-weighted images; the nidus demonstrates low to intermediate signal intensity, though on T2weighted images, the nidus intensity is increased $(9,10)$. The misleading appearance of osteoid osteoma on CT or MRI often leads to the diagnosis of osteomyelitis.

Although both the entities are quite similar in their clinical and radiological presentation, the incidence of brodie's abscess is lower than that of osteoid osteoma (7). However, some radiological features can help distinguish between the two. The presence of sinus tract on computed tomography is almost diagnostic for brodie's abscess. The nidus of osteoid osteoma is centrally placed in contrast to brodie's abscess where sequestration is 
eccentrically placed. Labeled scintigraphy has also been of use to distinguish the two but has been reported to be non-specific $(9,10)$.

This case re-emphasizes the fact that a detailed histological and microbiological work up is essential before subjecting the patient to definitive treatment. Microbial cultures of tissue specimen from brodie's abscess are inconsistently positive for any organism (3). A microbial growth is hardly seen in 50\% of cases (3). Cultures, when positive, usually grow Staphylococcus aureus, as in this case; some other organisms have also been reported $(2,3,7)$.

The unusual location of the abscess in the neck of femur often results in incomplete excision of the diseased tissue, which further leads to recurrence. Moreover, it has been recommended that abscess in the femoral neck approached from the side where they are closest to cortex to minimize bone resection (6). However, in this case, as the abscess was located in the calcar region, it was decided to approach it laterally, thereby avoiding any disruption in the medial calcar.

In the light of the above discussion, it can be concluded that brodie's abscess in femoral neck is a rare entity and can mimic osteoid osteoma clinico-radiologically. This case further substantiates the old dictum "culture every tumor and biopsy every infection before definitive treatment".

\section{REFERENCES}

1. Cabalena AE, Franklin H, Beabout JW. Osteomyelitis appearing as neoplasms. Arch Surg 1974; 109: 68-72.
2. Lindenbaum S,Alexander $H$. Infections simulating bone tumors. A review of subacute osteomyelitis. Clin Orthop 1984; 184: 193203.

3. Willis RB, Rozencwaig R. Pediatric osteomyelitis masquerading as skeletal neoplasia. Orthop Clin North Am 1996; 27: 625-634.

4. McGuinness B, Wilson N, Doyle AJ. The 'penumbra sign' on T1-weighted MRI for differentiating musculoskeletal infection from tumour. Skeletal Radiol 2007; 36: 417-21

5. King DM, Mayo KM. Subacute haematogenous osteomyelitis. J Bone Joint Surg [Br] 1969; 51-B: 458-63.

6. Stephens MM, MacAuley P. Brodie's abscess. A long term review. Clin Orthop 1988; 234: 211-216.

7. Abril JC, Castillo F, Casas J, Diaz A. Brodie's abscess of the hip simulating osteoid osteoma. Orthopedics 2000; 23: 285-287.

8. Gulati Y, Maheshwari AV. Brodie's abscess of the femoral neck simulating osteoid osteoma. Act Orthop Belg 2007; 73(5):648652.

9. Jaffe HL. Osteoid osteoma: a benign osteoblastic tumor composed of osteoid and atypical bone. Arch Surg 1935; 31: 709-728.

10. Mahboubi S. CT appearance of nidus in osteoid osteoma versus sequestration in osteomyelitis. J Comput Assist Tomogr 1986; 10: 457-459. 